\title{
Manifestações clínicas da síndrome de burnout em profissionais e estudantes de medicina: uma revisão bibliográfica
}

\author{
Clinical manifestations of professional burnout in professionals and medical students: a \\ bibliographic review
}
Manifestaciones clínicas del agotamiento profesional en profesionales y estudiantes de medicina: revisión bibliográfica

Isabhella Oliveira Marques Pio ${ }^{1 *}$, Alice Rodrigues Teixeira ${ }^{2}$, Brenda Barreto dos Santos ${ }^{3}$, Carla Fonseca Coelho², Hian Carlos Couto², João Inácio Pereira de Souza ${ }^{2}$, Mariana Nicolau Militão ${ }^{4}$, Gustavo Fonseca Genelhu Soares ${ }^{5}$.

\section{RESUMO}

Objetivo: Analisar e descrever as manifestações clínicas da Síndrome de Burnout nos profissionais e estudantes de medicina, levando em consideração sua abordagem física, psíquica e emocional, bem como avaliar as consequências dessa síndrome na vida profissional e pessoal do médico e de seus pacientes. Revisão bibliográfica: A partir de uma revisão narrativa sobre o tema, verificou-se a crescente prevalência da Síndrome de Burnout entre os estudantes de medicina e os profissionais médicos, agravada pelo momento atual de enfrentamento à pandemia do COVID-19. Evidenciou-se que a síndrome se caracteriza em três dimensões, a saber: por exaustão emocional, despersonalização e baixa realização profissional. Considerações finais: É de extrema importância que sejam desenvolvidas formas de enfrentamento, individuais e institucionais, com a finalidade de atenuar os problemas existentes no ambiente de trabalho, diminuir as dificuldades apresentadas, dar suporte aos trabalhadores, propiciar-lhes melhores condições de vida dentro e fora da instituição e, por consequência, melhorar a qualidade do cuidado prestado ao indivíduo.

Palavras-chave: Esgotamento profissional, Médicos, Diagnóstico.

\begin{abstract}
Objective: To analyze and disclose the clinical manifestations of Burnout in the medical professionals and medical students, taking into account its physical, psychological and emotional approach, as well as how to assess the consequences of the syndrome in professional and personal life of the doctor and his patients. Literature review: From a narrative review on the topic, there was an increasing prevalence of Burnout Syndrome among medical students and medical professionals, aggravated by the current moment of confrontation with the COVID-19 pandemic. It wasshown that the syndrome is characterized in three dimensions, namely: by emotional exhaustion, depersonalization and low professional realization. Final considerations: It is extremely important that ways of coping are developed, individual and institutional, with the aim of attenuating the existing problems in the work environment, reducing the difficulties presented, giving support to the workers, to promote the better living conditions in the institution and outside, consequently, to improve the quality of care provided to the individual.
\end{abstract}

Key words: Burnout, Professional, Physicians, Diagnosis.

\section{RESUMEN}

Objetivo: Analizar y describir las manifestaciones clínicas del Agotamiento Profesional en profesionales y estudiantes de medicina, teniendo en cuenta su abordaje físico, psíquico y emocional, así como evaluar las

${ }^{1}$ Centro Universitário do Espírito Santo (UNESC), Colatina - ES. *E-mail: isabhellamarques@hotmail.com

${ }^{2}$ Faculdade Vértice (Univértix), Matipó - MG.

${ }^{3}$ Faculdade Estácio Alagoinhas (FEA), Alagoinhas - BA.

${ }^{4}$ Faculdade de Minas (FAMINAS), Belo Horizonte - MG.

${ }^{5}$ Centro Universitário de Caratinga (UNEC), Caratinga - MG.

SUBMETIDO EM: 1/2021

ACEITO EM: 3/2021

PUBLICADO EM: $3 / 2021$ 
consecuencias de este síndrome en la vida profesional y personal del médico y sus pacientes. Revisión bibliográfica: a partir de una revisión narrativa sobre el tema, hubo una prevalencia creciente del síndrome de Burnout entre estudiantes de medicina y profesionales médicos, agravada por el momento actual de enfrentamiento a la pandemia de COVID-19. Se evidenció que el síndrome se caracteriza en tres dimensiones, a saber: por agotamiento emocional, despersonalización y bajo rendimiento profesional. Consideraciones finales: Es extremadamente importante que se desarrollen formas de afrontamiento, con el fin de mitigar los problemas existentes en el ambiente laboral, reducir las dificultades que se presentan, brindar apoyo a los trabajadores, brindándoles mejores condiciones de vida dentro y fuera de la institución y, en consecuencia, mejorando la calidad de la atención brindada al individuo.

Palabras clave: Agotamiento professional, Médicos, Diagnóstico.

\section{INTRODUÇÃO}

A Síndrome de Burnout é uma condição de alteração psicológica que geralmente acomete trabalhadores que realizam tarefas que necessitam lidar diretamente com outras pessoas e demandam alta responsabilidade, a exemplo dos médicos. Esse transtorno é caracterizado por três dimensões: exaustão emocional, despersonalização e baixa realização profissional (ROMANI M e ASHKAR K, 2014).

A exaustão emocional é caracterizada como a sensação pessoal de um profissional sentir-se incapaz de suprir as demandas que lhe são impostas, tal qual um sentimento de sobrecarga. A despersonalização, por sua vez, caracteriza-se como um distanciamento e desumanização do trabalhador em relação às pessoas do seu ambiente profissional, a exemplo frieza, indiferença e hostilidade. Por fim, a baixa realização pessoal está relacionada à baixa produtividade e insatisfação com o ambiente de trabalho e os serviços prestados (USECHE S, et al., 2017; MOL MC, et al., 2015).

A Síndrome de Burnout apresenta manifestações clínicas de amplo espectro, com repercussões físicas, psíquicas, emocionais ou comportamentais, cada uma contendo particularidades específicas de acordo com o seu foco. Entre os sintomas físicos, podem ser destacados problemas relacionados ao sono, como insônia e outros distúrbios; disfunções musculares: dores, fadiga e dificuldade de manter-se relaxado; cefaléia e transtornos gastrointestinais e alimentares. Os sintomas emocionais, por sua vez, apresentam-se por meio de irritabilidade, tristeza e desânimo, além da possibilidade de ocasionar transtornos de ansiedade e depressão. Em relação às manifestações psíquicas, destacam-se: confusão de pensamentos, dificuldade de concentração e falha ou diminuição de memória. Por fim, os indícios comportamentais sugerem falta de disposição e curiosidade, isolamento e distanciamento das atividades trabalhistas e/ou sociais (PEREIRA AB e GONÇALVES MB, 2009).

Entre os profissionais de saúde, sobretudo os médicos, a Síndrome de Burnout pode ocasionar consequências desde o âmbito ocupacional até o social, bem como afetar a qualidade de vida, influenciar na relação médico-paciente, diminuir a qualidade dos serviços prestados, autodepreciação, dependência de drogas lícitas e ilícitas, submissão à uma grande quantidade de medicamentos, além de afetar direta e indiretamente a instituição que o profissional atua, pois interfere na qualidade do trabalho e na relação com os colegas e o ambiente (SILVA RD, et al., 2018; CASTRO CA, et al., 2020; MOSS M, et al., 2017).

O estresse crônico tem sido um problema de considerável prevalência entre os médicos brasileiros nos últimos anos, principalmente devido à alta carga horária e demanda que estes precisam suprir (PAIVA LC, et al., 2017; LATORRACA CC, et al., 2019). No contexto que antecede à pandemia do Coronavirus Disease 2019 (COVID-19), causador da Síndrome Respiratória Aguda Grave 2 (SARS-CoV-2), os estudos epidemiológicos a respeito dessa síndrome no país mostravam-se, de certa maneira, escassos, sobretudo devido à recente definição do termo "Síndrome de Burnout". No entanto, quando comparados os períodos de pré e durante a pandemia, tornou-se notória a prevalência e o crescimento da síndrome no Brasil, o que pode ser explicado pelo trabalho desenvolvido na linha de frente de combate ao COVID-19 por profissionais da saúde, o que torna essa problemática uma preocupação pública (ORTIZ JM, et al., 2020).

Diante do exposto, o presente estudo tem como objetivo analisar e descrever as manifestações clínicas do transtorno de estresse agudo nos profissionais e estudantes de medicina, levando em consideração sua 
abordagem física, psíquica e emocional, bem como avaliar as consequências dessa síndrome na vida profissional e pessoal do médico e de seus pacientes.

\section{REVISÃO BIBLIOGRÁFICA}

A Classificação Estatística Internacional de Doenças e Problemas Relacionados à Saúde (CID-10) descreve clinicamente a Síndrome de Burnout como um estado de exaustão vital, fazendo parte da categoria "problemas relacionados à dificuldade de gerenciamento da vida" (KUHN CM e FLANAGAN EM, 2016). Em 1974, o psicólogo americano Herbert Freudenberger foi responsável por trazer pela primeira vez na literatura o conceito de Burnout, descrevendo a situação de muitos profissionais que, por meio do seu trabalho, experimentam exaustões emocionais, físicas, além do estresse e ansiedade (ROTHENBERGER DA, 2017).

A síndrome é separada em três dimensões: despersonalização, exaustão emocional e pouca realização pessoal no ambiente de trabalho. A despersonalização ocorre quando pacientes são tratados com indiferença por médicos, além do desenvolvimento de ações negativas acerca da profissão. A exaustão emocional tem como principal motivo a exposição prolongada ao estresse, se manifestando através da sensação de impotência, ausência de entusiasmo pelo trabalho. Já atitudes como o desligamento do trabalho ou o distanciamento do indivíduo de responsabilidades tem relação com a baixa realização profissional (WEST CP, et al., 2018; ROMANI M e ASHKAR K, 2014). Esse distúrbio apresenta evolução lenta e raramente é diagnosticado em seus estágios iniciais (PAIVA LC, et al., 2017).

A síndrome de Burnout se faz presente desde a faculdade, permanece durante a residência e, por fim, amadurece no cotidiano dos médicos. Nos Estados Unidos, cerca de $50 \%$ dos médicos em atividade relataram a presença de pelo menos um sintoma em algum momento ao decorrer de suas carreiras. No Canadá $12,5 \%$ dos médicos residentes pontuaram positivamente nas três dimensões juntas (ROMANI M e ASHKAR K, 2014; KUHN CM e FLANAGAN EM, 2016).

A prevalência da síndrome entre estudantes de medicina varia entre 31 e 49,6\%. Já em relação a residência médica, 50 e $76 \%$ dos residentes da área cirúrgica e medicina interna são afetados, respectivamente (ROMANI M e ASHKAR K, 2014). Na população em geral, níveis mais elevados de educação, incluindo graus profissionais, são fatores de proteção contra o Burnout, entretanto, esse risco aumenta quando se trata de uma graduação no curso de medicina (KUHN CM e FLANAGAN EM, 2016).

\section{Manifestações clínicas da síndrome e fatores associados}

O profissional acometido por essa doença pode vivenciar inúmeras alterações psíquicas, como lentidão do pensamento, dificuldade de concentração e déficit de memória. Além disso, disfunções emocionais e comportamentais também são evidenciadas em portadores da síndrome de Burnout, como irritabilidade, ansiedade, alienação, desânimo, comportamento egocêntrico, perda da iniciativa, negligência, tendência ao isolamento e desinteresse (BOND MK, et al., 2018).

Tal esgotamento profissional também se manifesta com sintomas físicos. Dores musculares, fadiga constante, cefaleia intensa e distúrbios do sono podem estar presentes nesse distúrbio. Além disso, alterações intestinais, como úlceras e gastrite, e afecções dermatológicas, como alergias e alopécia, são manifestações possíveis da doença. Os sistemas cardiovascular e respiratório também são passíveis de acometimento, podendo o profissional apresentar risco elevado para o surgimento de hipertensão arterial sistêmica, doença coronariana, asma e bronquite (BOND MK, et al., 2018; TRIGO TR, et al., 2007). Outras repercussões clínicas da síndrome envolvem alterações do ciclo menstrual nas mulheres e disfunção sexual em ambos os sexos, com queda da libido, impotência sexual, ejaculação precoce e dispareunia. Há também o aumento da suscetibilidade a novas infecções devido à imunodeficiência causada por essa condição (TRIGO TR, et al., 2007).

As consequências de numerosos fenômenos clínicos têm impacto negativo na saúde física e mental dos profissionais de saúde. Observa-se diminuição na qualidade e rendimento do trabalho, aumento da realização de procedimentos equivocados e a presença de imprudência e negligência no ambiente profissional. No âmbito pessoal, o Burnout pode provocar no indivíduo o distanciamento dos familiares e colegas. Ademais, a 
síndrome está associada ao aumento do abuso e dependência de substâncias lícitas e ilícitas, como tabaco, álcool e benzodiazepínicos, e também a depressão e autoextermínio, com o aumento de ideações e tentativas suicidas (TRIGO TR, et al., 2007).

\section{Relação com o momento atual da pandemia do Coronavirus Disease 2019 (COVID-19)}

Como já citado, a Síndrome de Burnout é um problema crescente entre profissionais de saúde, no entanto, antes da pandemia do Coronavirus Disease 2019 (COVID-19), causador da Síndrome Respiratória Aguda Grave 2 (SARS-CoV-2), estudos epidemiológicos sobre esse tema no Brasil eram escassos. Contudo, estimase que haverá um aumento progressivo deste transtorno no país (RIBEIRO LM, et al., 2020; MACÍARODRÍGUEZ C, et al., 2020).

A enfermidade tem presença marcante durante a pandemia, estabelecendo um problema de saúde pública por afetar profissionais de saúde e servidores gerais das entidades de saúde que permanecem na linha de frente de combate ao COVID-19, entre eles os médicos (ORTIZ JM, et al., 2020). Segundo Brooks SK, et al. (2020) os sistemas de saúde de muitos países atingidos entraram em decadência e os profissionais de saúde se tornam cada vez mais exaustos e afetados com demanda aumentada de trabalho e o distanciamento social, que é o método de controle mais efetivo e adotado para reduzir a disseminação da doença.

Os profissionais médicos enfrentam, além do risco de contaminação e demais riscos ocupacionais inerentes à exposição ao vírus, situações como a falta de recursos, turnos de trabalho mais longos, interrupções no sono e desequilíbrio entre trabalho e vida pessoal (ADAMS JG, WALLS RM, 2020). Nesse contexto, o profissional tende a desenvolver um desgaste físico e emocional, além do surgimento de problemas como: hipertensão arterial, náuseas, estresse, doenças entéricas, esgotamento mental, depressão, exaustão e sono prejudicado (BROOKS SK, et al., 2020)

O estabelecimento da Síndrome de Burnout se tornou ainda mais propício com a COVID-19, visto que a situação da saúde no Brasil já era desfavorável e agravou-se na pandemia, tornando-se um importante problema de saúde pública no país. Dessa forma, torna-se essencial o desenvolvimento e reforço das estratégias destinadas a fortalecer a saúde mental dos profissionais afetados (ORTIZ JM, et al., 2020; MACÍARODRÍGUEZ C, et al., 2020).

\section{Estratégias de tratamento para a Síndrome de Burnout}

Indubitavelmente, a Síndrome de Burnout traz consequências indesejáveis tanto para o profissional, quanto para o paciente e a instituição onde esse profissional atua. Por isso, é de extrema importância que sejam desenvolvidas formas de enfrentamento com a finalidade de atenuar os problemas existentes no ambiente de trabalho, diminuir as dificuldades, dar suporte aos trabalhadores, propiciar-lhes melhores condições de vida dentro e fora da instituição e, por consequência, melhorar a qualidade do cuidado prestado ao indivíduo.

As estratégias para o enfrentamento dessa síndrome variam de acordo com o objetivo desejado. As intervenções focadas no indivíduo, por exemplo, são baseadas em habilidades comportamentais e cognitivas de coping, meditação, educação em saúde e atividade física. Tem-se, ainda, estratégias baseadas na relação indivíduo-instituição, compreendendo as ações para melhoria da comunicação e trabalho em equipe. Há também estratégias baseadas na organização, como treinamento e mudança das condições físico-ambientais e, por fim, intervenções combinadas que associam dois ou mais tipos de intervenções com focos distintos (MURTA SG, et al., 2007). Outros estudos também enfatizam os três níveis de intervenções: centrados na resposta do indivíduo (individual), no contexto ocupacional (organizacional) e na interação contexto ocupacional e indivíduo (combinadas) (MORENO FN, et al., 2011).

As estratégias individuais consideram as características pessoais e respostas emocionais frente a uma situação estressante. Os programas baseados nessas características consistem basicamente na aprendizagem, por parte do profissional, de estratégias de enfrentamento adaptativas diante de agentes estressantes, conseguindo prevenir as respostas negativas associadas aos efeitos do estresse. Evidencia-se que o profissional de saúde que se sente exausto pelas relações do trabalho, presta pouca atenção para sua 
qualidade de vida e saúde mental, ignorando suas necessidades e os recursos disponíveis para melhorar sua vida laboral, e consequentemente seu bem-estar individual (MORENO FN, et al., 2011).

Dessa forma, uma das estratégias que pode ser utilizada como forma de prevenção no desenvolvimento da síndrome é o coping, que é definido como "esforço cognitivo e comportamental realizado para dominar, tolerar ou reduzir as demandas internas e externas". O coping pode ser dividido em duas categorias funcionais: o coping focalizado na emoção e o coping focalizado no problema. No primeiro, há procura de estratégias que derivam de processos defensivos como evitar distanciar-se e buscar valores positivos em eventos negativos, como forma de diminuir a importância emocional do estressor. Já o coping focalizado no problema baseia-se na tentativa de definição da dificuldade vivenciada, procurando soluções alternativas e optando entre elas (RODRIGUES AB, 2006).

Além do coping, reforça-se que adquirir hábitos de vida saudáveis, como praticar exercícios físicos regularmente, dormir bem, manter uma dieta equilibrada e usufruir do lazer são necessários para diminuir os efeitos do estresse profissional. As pessoas que se exercitam regularmente relatam frequentemente um efeito de bem-estar (USHER K, et al., 2016). A atividade física traz benefícios para indivíduos com sintomas ansiosos e de estresse, melhorando a qualidade de vida dessas pessoas. Resultados de estudos indicam que o estresse possui forte influência no estado de ansiedade para esses indivíduos. Isto sugere que os profissionais de saúde devem transformar a prática de atividade física em um hábito, como estratégia benéfica para gerenciamento do estresse (MELO LC, et al., 2014).

Já a estratégias centradas no contexto ocupacional enfatizam a necessidade de modificar a situação em que se desenvolvem as atividades, principalmente no âmbito organizacional, tais como ambiente e clima de trabalho (MORENO FN, et al., 2011). Alguns autores consideram que a Síndrome de Burnout não é um problema das pessoas, mas principalmente dos lugares onde elas atuam. Logo, quando a organização do trabalho se estrutura de forma rígida, valorizando somente o aspecto econômico, o resultado será um desajuste entre o trabalhador e processo de trabalho, empobrecendo a versatilidade psíquica do profissional e abrindo a possibilidade para a descompensação da saúde mental (FERNANDES JD, et al., 2006).

Dessa forma, a maneira como o trabalho tem sido organizado necessita ser revista, com ações modificadoras que promovam o bem-estar e previnam o surgimento de doenças, com medidas que se iniciam na cultura institucional até às condições de trabalho, com recursos humanos suficientes, disponibilidade de materiais, autonomia, participação na tomada de decisão, para realização da atividade com satisfação, resolução de conflitos de forma imparcial e justa. Para atender às exigências da instituição, o profissional deve se adequar às formas de trabalho, para isso, a organização também deve ser flexível para facilitar este processo (GIL GP, et al., 2010).

Por fim, programas com estratégias combinadas, centrados na interação do contexto ocupacional e o indivíduo têm como objetivo entender o Burnout como resultante da relação do sujeito e o meio laboral, evidenciando de forma integrada as modificações das condições de trabalho, a percepção do trabalhador e o modo de enfrentamento diante das situações de estresse (MORENO FN, et al., 2011).

Nesse contexto, é necessário que se desenvolvam ações preventivas como reuniões de equipe para discussões e reflexões dos problemas. Sugerem-se, ainda, palestras e programas que informem os profissionais quanto aos riscos a que estão expostos e a identificação das manifestações da síndrome. Dessa forma, essas estratégias coletivas buscam a manutenção do equilíbrio mental e físico diante das ameaças do ambiente do trabalho e são relevantes pois proporcionam a integração da equipe, promovem a troca de experiência e apoio mútuo e reduzem a tensão no ambiente de trabalho (HADDAD ML, 2010).

\section{CONSIDERAÇÕES FINAIS}

Diante do exposto, a crescente prevalência da Síndrome de Burnout entre os estudantes de medicina e entre os profissionais médicos, agravada pelo momento atual de enfrentamento à pandemia do COVID-19, evidencia a necessidade de desenvolvimento e reforço das estratégias destinadas a fortalecer a saúde mental dos profissionais afetados. Dessa forma, é de extrema importância que sejam desenvolvidas formas de 
enfrentamento com a finalidade de atenuar os problemas existentes no ambiente de trabalho, diminuir as dificuldades, dar suporte aos trabalhadores, propiciar-lhes melhores condições de vida dentro e fora da instituição e, por consequência, melhorar a qualidade do cuidado prestado ao indivíduo.

\section{REFERÊNCIAS}

1. ADAMS JG, WALLS RM. Supporting the health care work force during the COVID-19 global epidemic. JAMA, 2020; 232(15): 1439-1440.

2. BOND MK, et al. Prevalência de Burnout entre médicos residentes de um hospital universitário. Revista Brasileira de Educação Médica, 2018; 43(3): 97-107.

3. BROOKS SK, et al. The psychological impact of quarantine and how to reduce it: rapid review of the evidence. The Lancet, 2020; 395(102227): 912-920.

4. CASTRO CA, et al. Síndrome de burnout e engajamento em profissionais de saúde: um estudo transversal. Revista Brasileira de Terapia Intensiva, 2020; 32(3): 381-390.

5. FERNANDES JD, et al. Saúde mental e trabalho: significados e limites de modelos teóricos. Revista Latino-Americana de Enfermagem. 2006. 14:803-811.

6. GIL GP, et al. Estresse do enfermeiro em unidade de emergência. Revista Latino-Americana de Enfermagem, 2010; 14:534-539.

7. HADDAD ML, et al. Riscos psicossociais relacionados ao trabalho das equipes de saúde da família: percepção dos profissionais. Revista de Enfermagem da UFRJ, 2010; 15:502-507.

8. KUHN CM, FLANAGAN EM. Self-care as a professional imperative: physicianburnout, depression, and suicide. Canadian Journal of Anaesthesia, 2017;64(2):158-168

9. LATORRACA CC, et al. O que as revisões sistemáticas Cochrane dizem sobre prevenção e tratamento da síndrome de burnout e estresse no trabalho. Diagnóstico e Tratamento, 2019; 24(3): 119-125.

10. MACÍA-RODRÍGUEZ C, et al. Burnout syndrome in internal medicine specialists and factors associated with its on set. Revista Clinica Espanola, 2020; 220(6): 331-338.

11. MELO LC, et al. A educação física no âmbito do tratamento em saúde mental: um esforço coletivo e integrado. Revista Latino-americana Psicopatologia Fundamental, 2014; 17(3):501-514.

12. MOL MC, et al. The Prevalence of Compassion Fatigue and Burnout among Health care Professionals in Intensive Care Units: A Systematic Review. PLOS ONE, 2015; 10(8): e0136955.

13. MORENO FN, et al. Estratégias e intervenções no enfrentamento da Síndrome de Burnout. Revista de Enfermagem, $2011 ; 19(1): 140-145$

14. MOSS M, et al. An Official Critical Care Societies Collaborative Statement: Burnout Syndrome in Critical Care Health care Professionals: A Call for Action, 2016; 150(1):17-26.

15. MURTA SG, et al. Stress ocupacional em bombeiros: efeitos de intervenção baseada em avaliação de necessidades. Estudos de Psicologia, 2007; 24(1): 41-51.

16. ORTIZ JR, et al. Consecuencias de la pandemia covid-19 enlasalud mental asociadas al aislamiento social. Asociación Mexicana de Psicoterapia y Educación, 2020: 1-21

17. PAIVA LC, et al. Burnout syndrome in health-care professionals in a university hospital. Clinics, 2017; 72(5): $305-309$.

18. PEREIRA AB e GONÇALVES MB. Transtornos emocionais e a formação em Medicina: um estudo longitudinal. Revista Brasileira de Educação Médica, 2009; 33(1): 10-23.

19. RIBEIRO LM, et al. Síndrome de burnout em profissionais de saúde antes e durante a pandemia da COVID-19. Revista Eletrônica Acervo Saúde, 2020; 12(11):1-10.

20. RODRIGUES AB. Burnout e estilos de coping de enfermeiros que assistem pacientes oncológicos. Tese (Doutorado em Enfermagem) - Escola de Enfermagem. Universidade de São Paulo, São Paulo, 2006; 169p.

21. ROMANI M, ASHKAR K. Burnout among physicians. The Libyan journal of medicine,2014;9:23556.

22. ROTHENBERGER DA. Physician Burnout and Well-Being: A Systematic Review and Framework for Action. Diseases of the colon and rectum, 2017; 60(6):567-576.

23. SILVA RD, et al. Síndrome de Burnout: realidade dos fisioterapeutas intensivistas? Fisioterapia e Pesquisa, 2018; 25(4): 388-394.

24. TRIGO TR, et al. Síndrome de Burnout ou estafa profissional e os transtornos psiquiátricos. Revista de Psiquiatria Clínica, 2007; 34(5): 223-233.

25. USECHE S, et al. Comparing Job Stress, Burnout, Health and Traffic Crashes of Urban Bus and BRT Drivers. American Journal of Applied Psychology, 2017;5(1): 25-32.

26. USHER K, et al. A randomized controlled trial under taken to test a nurse-led weight management and exercise intervention designed for people with serious mental illness who take second generation antipsychotics. Journal of Advanced Nursing, 2013; 69(7):1539-1548.

27. WEST CP, et al. Physicianburnout: contributors, consequences and solutions. Journal of internal medicine, 2018; 283(6):516-529. 